\title{
Progression of cardiovascular autonomic neuropathy and cardiovascular disease in type 2 diabetes
}

Jae-Seung Yun ${ }^{1}$, Yong-Moon Park², Seon-Ah Cha', Yu-Bae Ahn ${ }^{1}$ and Seung-Hyun Ko ${ }^{1 *}$ (D)

\begin{abstract}
Background: To examine whether the progression rate of cardiovascular autonomic neuropathy (CAN) stage is an independent predictive factor for cardiovascular disease (CVD) in type 2 diabetes.

Methods: Standardized cardiovascular autonomic reflex tests (CARTs) using traditional Ewing method were performed at baseline. The follow-up CARTs was recommended once every two years. We estimated the primary CVD endpoint, defined as coronary artery disease and ischemic stroke. The association between the progression rate of CAN stage and CVD was examined using time-dependent Cox proportional hazard models.
\end{abstract}

Results: At baseline, 578 patients completed follow-up CARTs; the cohort comprised 329 women (56.9\%) with a mean age of $58.3 \pm 10.3$ years and a mean diabetes duration of $10.1 \pm 6.2$ years. One hundred and seventy-six patients (30.4\%) developed CAN progression between baseline and follow-up CARTs. In the multivariable Cox proportional hazards regression analysis, patients with CAN progression demonstrated a 3.32 times higher risk ( $95 \%$ confidence interval, $C l 1.81-6.14, P<0.001)$ of CVD than those without CAN progression. Patients who experienced CAN progression from the normal to definite stage had the greatest risk of CVD compared to other patients (hazard ratio 4.91, 95\% $\mathrm{Cl} 2.05-11.77$, P for trend $=0.001$ ).

Conclusions: CAN stage progression was associated with an increased risk of CVD in this type 2 diabetes cohort. Patients with rapid CAN progression had the greatest risk of CVD. Thus, regular screening and risk management of CAN progression is necessary to prevent CVD.

Keywords: Cardiovascular autonomic neuropathy, Cardiovascular disease, Type 2 diabetes

\section{Background}

Cardiovascular autonomic neuropathy (CAN) is one of the most common and serious complications associated with diabetes and is defined as the impairment of the autonomic control of the cardiovascular system [1]. CAN is caused by damage to the autonomic nerve fibers that innervate the heart and blood vessels, leading to abnormal control of heart rate and cardiac performance [2]. Clinical manifestations of CAN are resting tachycardia,

\footnotetext{
${ }^{*}$ Correspondence: kosh@catholic.ac.kr

${ }^{1}$ Division of Endocrinology and Metabolism, Department of Internal Medicine, St. Vincent's Hospital, College of Medicine, The Catholic University of Korea, Ji-dong, Paldal-gu, Suwon 16247, South Korea Full list of author information is available at the end of the article
}

exercise intolerance, orthostatic hypotension, and silent myocardial infarction [3], which can affect the daily activities and quality of life of patients with diabetes and may lead to life-threatening outcomes [4]. Thus, CAN assessment is important for establishing a strategy for diabetes care and for predicting the prognosis of patients with diabetes.

CAN is a progressive disease entity $[3,5]$ that is considered to progress from a subclinical stage, characterized by abnormalities of heart rate variability, to a clinically apparent stage with diverse and disabling symptoms [5]. Risk factors associated with CAN include hyperglycemia, duration of diabetes, hypertension, dyslipidemia, and obesity in type 2 diabetes [6]. Cardiovascular autonomic reflex tests (CARTs) are the most commonly used 
methods for the diagnosis of CAN and can easily assess cardiovascular autonomic function based on heart rate response to deep breathing, Valsalva maneuver, and postural change $[7,8]$. Generally, the presence of CAN is associated with poor outcomes among diabetes complications [1, 9-12].

Several previous studies suggested that CAN contributes to an increased risk of cardiovascular disease (CVD) and CVD-related mortality. Heart rate variability is a well-known predictive factor of silent myocardial infarction, recurrent CVD, and post-myocardial infarction mortality [13-15]. However, no study has evaluated the association between the progression of CAN and cardiovascular events among patients with type 2 diabetes. We hypothesized that a more rapid CAN progression is related to poorer outcomes of cardiovascular events. This study aimed to evaluate the association between the progression of CAN and CVD using standardized CARTs in patients with type 2 diabetes.

\section{Methods \\ Participants}

This prospective study was approved by the Ethics Committee of the Catholic Medical Center and was performed according to the Declaration of Helsinki. All participants provided signed written informed consent. From 2000 to 2008, 1073 patients with type 2 diabetes were included and underwent baseline CARTs at the University-affiliated Diabetes Center of St. Vincent's Hospital in South Korea. Patients were excluded if they had a history of CVD and any form of severe diseases such as: severe infection, liver cirrhosis, malignancy, or heart failure. Patients with definite CAN (autonomic function test score $\geq 2$ ) or arrhythmia (e.g., atrial fibrillation) were also excluded at the initial visit and follow-up, which is the second test period. The study design summary is shown in Additional file 1: Figure S1.

\section{CAN evaluation}

CARTs were performed using the standardized Ewing method with the Monitor One nDX device (QMed Inc., Eatontown, NJ). CARTs included a test of heart rate variability, including the $\mathrm{R}-\mathrm{R}$ response to paced breathing (expiration/inspiration ratio, E/I ratio), Valsalva maneuver, and postural change from lying to standing, as previously described [6]. Patients were asked to fast for $12 \mathrm{~h}$ before the autonomic function test and to avoid taking insulin, anti-depressants, neuroleptic agents, caffeine, nicotine, antihistamines, or sympatholytic drugs that could affect the results of the cardiovascular autonomic test [7]. E/I ratios below the age-related reference value, Valsalva ratios $<1.20$, and posture ratios $<1.03$ were considered abnormal [16]. Each of the ratios was calculated as normal (0) or abnormal (1), with a maximum total score of 3 . A CAN stage score of 0 was defined as a normal autonomic function, whereas scores of 1 and 2 or more were defined as early CAN and definite CAN, respectively [6]. The follow-up CARTs was recommended once every two years unless there were any specific CAN-related symptoms within 1 year following baseline CARTs. CAN progression was defined as an increased score in the follow-up test compared with the score in the baseline test. The progression group was categorized into the following 4 subgroups based on the status at baseline and follow-up CAN stages: non-progression, normal to early stage, early to definite stage, and normal to definite stage.

\section{Data collection}

At follow-up, second autonomic function test, patient height, weight, and blood pressure were measured. Hypertension was defined as systolic blood pressure $\geq 140 \mathrm{mmHg}$, diastolic blood pressure $\geq 90 \mathrm{mmHg}$, or any use of antihypertensive medications. All laboratory measurements were performed after a 12-h overnight fast. Fasting plasma glucose levels were measured using an automated enzymatic method, with HbA1c levels determined using high-performance liquid chromatography. Blood lipid concentrations of total cholesterol, triglycerides, HDL-cholesterol, and LDL-cholesterol levels were measured enzymatically using an automatic analyzer. HbA1c levels were measured at least once every 6 months, and lipid profiles were measured at least once each year to evaluate glycemic control. Mean HbA1c and mean lipid profiles were calculated as the average $\mathrm{HbA1c}$ and lipid profile levels during the period from baseline CARTs to follow-up CARTs. These were used to adjust the baseline glycemic status in this analysis (Additional file 1: Figure S1). Urinary albumin excretion was measured via immunoturbidimetry (Eiken, Tokyo, Japan) with 24-h urine collection at baseline. To determine renal function, patients' serum creatinine and eGFR levels were assessed. Additionally, a kinetic picrate method (Jaffe method) was used to determine serum creatinine levels. The most recent Chronic Kidney Disease Epidemiology Collaboration equations were used to calculate patients' eGFR and measure the serum creatinine level [17].

\section{Cardiovascular outcomes}

Patients who underwent follow-up second CARTs received follow-up clinical care until December 2016 (Additional file 1: Figure S1). The primary endpoint was the development of the first cardiovascular event. CVD was defined as a diagnosis of coronary artery disease or stroke. Coronary artery disease included angina 
pectoris, myocardial infarction, or coronary revascularization (coronary bypass, surgery, or coronary angioplasty) [18]. Stroke history included a previous transient ischemic attack or cerebral infarction [19]. We verified the onset of CVD via interviews at every visit and by using medical records. A diagnosis of a cardiovascular event was confirmed by specialists, including a cardiologist, neurologist, and neurosurgeon. The cause and time of death were obtained from hospital records or by making regular telephone call checks when patients did not attend a follow-up.

\section{Statistical analysis}

Baseline characteristics are presented as means and standard deviations or medians with interquartile ranges. Chi square tests were used to assess the differences in the proportion of categorical variables. Independent Student $t$-tests were used for evaluating differences between the means of 2 continuous variables. Kaplan-Meier plots were used to illustrate the cumulative incidence of the first cardiovascular event according to CAN progression. We used Cox proportional hazard regression analysis to assess the associations between outcomes and potential explanatory variables. Proportional hazards assumptions were examined using log-minus log-survival plots. There was no significant departure from proportionality in hazards over time. A time-dependent Cox proportional hazards regression model was used to identify associations between CAN and CVD, with CAN progression considered the time-dependent variable. Potential confounders were identified a priori based on literature reviews. The first model was the crude model; the second was the age and sex-adjusted model. The third model was analyzed after adjusting for the following risk factors: sex, age, duration of diabetes, presence of hypertension, body mass index (BMI), smoking, alcohol consumption, use of medications [insulin, ACE inhibitor, angiotensin II receptor blocker (ARB), aspirin, statin], mean $\mathrm{HbA1c}$, standardized deviation of $\mathrm{HbA1c}$ levels, and mean LDL-cholesterol level between first assessment and follow-up CARTs period, urinary albumin excretion rate, and estimated GFR. We performed an additional analysis, which included the group with definite CAN at initial CARTs, in order to compare CVD risk between the group with definite CAN during initial CARTs and that with CAN progression. The associations between CAN progression and other variables were examined to determine the effect modification in this model. When $P$ for an interaction was significant we performed stratified subgroup analysis. Statistical analyses were performed using SAS version
9.3 (SAS Institute Inc., Cary, NC, USA). $P$ value, $P$ for trend, and $P$ for interaction were considered statistically significant at $<0.05$.

\section{Results}

In this study, 174 patients (30.1\%) had CAN progression between baseline and follow-up CARTs. The mean duration between the two assessments was 2.3 years. The screening intervals between baseline and followup CARTs were not different between the CAN stage groups (Additional file 2: Table S1). Patients with CAN progression were older; had a longer duration of diabetes; used more insulin; and had a higher level of HbA1c, fasting plasma glucose, LDL-cholesterol, and urinary albumin excretion (Table 1). Of patients with CAN progression, progression from normal to early CAN was diagnosed in 79 patients (45.4\%), progression from early to definite CAN in 65 (37.3\%), and progression from normal to definite CAN in $30(17.2 \%)$. The patients who progressed from the normal to definite CAN stage had a higher fasting plasma glucose level $(9.4 \pm 1.5 \mathrm{mmol} / \mathrm{L}$ vs. $8.4 \pm 2.9 \mathrm{mmol} / \mathrm{L}, P<0.001)$, mean HbA1c $[9.2 \pm 1.4 \%(77.4 \pm 15.6 \mathrm{mmol} / \mathrm{L})$ vs. $8.3 \pm 1.5 \%$ $(67.0 \pm 16.0 \mathrm{mmol} / \mathrm{L}), P<0.001)$, and mean LDL-cholesterol level $(3.2 \pm 0.6 \mathrm{mmol} / \mathrm{L}$ vs. $2.7 \pm 0.8 \mathrm{mmol} / \mathrm{L}$, $P=0.004)$ compared with other patients who did not progress from the normal to definite CAN stage. However, there were no differences in age, duration of diabetes, presence of hypertension, BMI, smoking, alcohol consumption, use of medications, estimated GFR, and albumin excretion rate between the subgroups (Table 2). Among patients who progressed from normal to definite CAN, $86.7 \%$ had a score of $2,93.3 \%$ had an abnormal Valsalva ratio score, and $83.3 \%$ showed an abnormal posture ratio score. There was no difference in the interval duration of CARTs (from baseline to follow-up CARTs) between the subgroups $(P=0.126)$.

The median time for follow-up was 7.3 years. During the study period, a CVD event occurred in 55 patients (9.3\%). The overall incidence rate of a CVD event was 1.27 per 100 patient-years. Patients with CVD were older, had longer diabetes duration, used more insulin, and had a higher level of HbA1c at baseline, as well as lower eGFR levels (data not shown). The incidence of CVD increased in the group with CAN progression. This group demonstrated the highest rate of progression from normal to definite CAN. In the multivariate Cox proportional hazards regression analysis, patients with CAN progression had a 3.32 times higher risk of CVD than those without CAN progression. Patients who progressed from normal to definite CAN during the study period had a greatest risk of CVD than those of other patients following adjustment for potential 
Table 1 Baseline parameters between the group with and without progression of cardiovascular autonomic neuropathy

\begin{tabular}{|c|c|c|c|c|}
\hline & $\begin{array}{l}\text { Total } \\
(\mathrm{N}=578)\end{array}$ & $\begin{array}{l}\text { CAN progression }(-) \\
(\mathrm{N}=404)\end{array}$ & $\begin{array}{l}\text { CAN progression }(+) \\
(\mathrm{N}=174)\end{array}$ & $P$-value \\
\hline Women, n (\%) & $329(56.9)$ & $220(54.5)$ & $109(62.6)$ & 0.083 \\
\hline Age (years) & $58.3 \pm 10.3$ & $57.1 \pm 10.1$ & $60.9 \pm 10.3$ & $<0.001$ \\
\hline Diabetes duration (years) & $10.1 \pm 6.2$ & $9.1 \pm 5.7$ & $12.4 \pm 6.7$ & $<0.001$ \\
\hline Body mass index $\left(\mathrm{kg} / \mathrm{m}^{2}\right)$ & $24.7 \pm 3.2$ & $24.8 \pm 3.2$ & $24.7 \pm 3.4$ & 0.710 \\
\hline Hypertension, n (\%) & $242(43.8)$ & $163(42.2)$ & $79(47.6)$ & 0.284 \\
\hline Smoking, n (\%) & $124(21.5)$ & $93(23.0)$ & $31(17.8)$ & 0.198 \\
\hline Alcohol, n (\%) & $138(23.9)$ & $105(26.0)$ & $33(19.0)$ & 0.087 \\
\hline Insulin, n (\%) & $141(24.4)$ & $87(21.5)$ & $54(31.0)$ & 0.020 \\
\hline ACE inhibitor/ARBs, n (\%) & $181(31.3)$ & $122(30.2)$ & $59(33.9)$ & 0.433 \\
\hline Calcium channel blocker, n (\%) & $101(17.5)$ & $63(15.6)$ & $38(21.8)$ & 0.090 \\
\hline Aspirin, n (\%) & $45(7.8)$ & $28(6.9)$ & $17(9.8)$ & 0.318 \\
\hline Statin, n (\%) & $65(11.2)$ & $44(10.9)$ & $21(12.1)$ & 0.789 \\
\hline $\mathrm{FPG}(\mathrm{mmol} / \mathrm{L})$ & $8.4 \pm 2.9$ & $8.3 \pm 2.7$ & $8.8 \pm 3.3$ & 0.053 \\
\hline $\mathrm{eGFR}\left(\mathrm{mL} / \mathrm{min} / 1.73 \mathrm{~m}^{2}\right)$ & $85.6 \pm 17.1$ & $86.7 \pm 16.1$ & $83.3 \pm 19.0$ & 0.040 \\
\hline Mean HbA1c (\%) & $8.3 \pm 1.5$ & $8.1 \pm 1.5$ & $8.8 \pm 1.6$ & $<0.001$ \\
\hline SD HbA1c & $1.1 \pm 1.0$ & $1.1 \pm 1.0$ & $1.0 \pm 1.0$ & 0.787 \\
\hline Mean HbA1c (mmol/L) & $67.1 \pm 16.7$ & $65.1 \pm 16.0$ & $71.5 \pm 17.6$ & $<0.001$ \\
\hline Mean total cholesterol (mmol/L) & $4.7 \pm 0.9$ & $4.7 \pm 0.9$ & $4.8 \pm 0.9$ & 0.078 \\
\hline Mean triglyceride $(\mathrm{mmol} / \mathrm{L})$ & $1.7 \pm 1.0$ & $1.7 \pm 1.0$ & $1.7 \pm 0.9$ & 0.931 \\
\hline Mean HDL-cholesterol (mmol/L) & $1.2 \pm 0.3$ & $1.2 \pm 0.3$ & $1.1 \pm 0.3$ & 0.204 \\
\hline Mean LDL-cholesterol (mmol/L) & $2.8 \pm 0.8$ & $2.7 \pm 0.8$ & $2.9 \pm 0.8$ & 0.021 \\
\hline UAE (mg/day) & $79.8 \pm 298.9$ & $48.1 \pm 191.4$ & $153.8 \pm 453.3$ & 0.004 \\
\hline
\end{tabular}

Values are presented as number (\%) or mean \pm SD

CAN cardiovascular autonomic neuropathy, $A R B$ angiotensin receptor blocker, FPG fasting plasma glucose, eGFR estimated glomerular filtration rate, SD standard deviation, UAE urinary albumin excretion

confounders (Table 3, Fig. 1). We performed an additional analysis after including the group with definite CAN at baseline CARTs. The group with CAN progression (both early to definite and normal to definite) showed a higher risk for CVD compared to the group with maintained definite CAN (Additional file 2: Tables S2 and S3).

We analyzed the association between CAN progression and the risk of CVD within subgroups stratified by sex, age, diabetes duration, BMI, hypertension, HbA1c, and LDL-cholesterol. The association between CAN progression and CVD increased in participants aged $<60$ years, with a diabetes duration $<10$ years, a $\mathrm{BMI}<25 \mathrm{~kg} / \mathrm{m}^{2}$, and a mean HbA1c $>9 \%$ ( $P$ for interaction $<0.001)$. In contrast, sex, presence of hypertension, and mean LDL-cholesterol were not significantly associated with CAN progression and CVD (Table 3). The results of the sensitivity analyses after excluding patients who developed CVD within 2 years did not alter the significance of the association between CAN progression and CVD development (Table 3). During the follow-up period, 18 patients (3.1\%) died, of which $5(0.9 \%)$ died owing to CVD. The overall mortality rate was significantly higher in patients with CAN progression $(6.9 \%$ vs. $1.5 \%, P<0.001)$.

\section{Discussion}

In this prospective study, we demonstrated a significant association between CAN progression and CVD risk in patients with type 2 diabetes. Notably, patients with rapid CAN progression had the highest risk of CVD.

\section{Risk factors of CAN progression}

The progression and natural history of CAN are not completely understood. Findings from recent studies suggest some of the associated factors for CAN. Serum average and variability of glycated albumin level, which represent the short-term (2-3 weeks) glycemic status, are significantly associated with the presence of CAN [20]. Shimabukuro et al. demonstrated that short-term suppression of glycemic variability with $\alpha$-glucosidase inhibitor can improve sympathetic nervous system activity and modify 
Table 2 Descriptive characteristics according to the progression status of cardiovascular autonomic neuropathy

\begin{tabular}{|c|c|c|c|c|c|}
\hline & $\begin{array}{l}\text { Non-progression } \\
(\mathrm{N}=404)\end{array}$ & $\begin{array}{l}\text { Normal } \rightarrow \text { early } \\
(\mathrm{N}=79)\end{array}$ & $\begin{array}{l}\text { Early } \rightarrow \text { definite } \\
(\mathrm{N}=65)\end{array}$ & $\begin{array}{l}\text { Normal } \rightarrow \text { definite } \\
(\mathrm{N}=30)\end{array}$ & $P$-value \\
\hline Women, n (\%) & $220(54.5)$ & $49(62.0)$ & $41(63.1)$ & $19(63.3)$ & 0.341 \\
\hline Age (years) & $57.1 \pm 10.1$ & $59.9 \pm 10.0$ & $62.0 \pm 11.1$ & $60.7 \pm 8.8$ & $<0.001$ \\
\hline Diabetes duration (years) & $9.1 \pm 5.7$ & $11.4 \pm 6.6$ & $14.2 \pm 7.1$ & $11.5 \pm 5.6$ & $<0.001$ \\
\hline Body mass index $\left(\mathrm{kg} / \mathrm{m}^{2}\right)$ & $24.8 \pm 3.2$ & $24.2 \pm 3.4$ & $24.7 \pm 3.4$ & $25.7 \pm 2.9$ & 0.518 \\
\hline Hypertension, n (\%) & $163(42.2)$ & $35(46.7)$ & $31(50.0)$ & $13(44.8)$ & 0.655 \\
\hline Smoking, n (\%) & $93(23.0)$ & $12(15.2)$ & $15(23.1)$ & $4(13.3)$ & 0.295 \\
\hline Alcohol, n (\%) & $105(26.0)$ & $18(22.8)$ & $13(20.0)$ & $2(6.7)$ & 0.091 \\
\hline Insulin, n (\%) & $87(21.5)$ & $14(17.7)$ & $30(46.2)$ & $10(33.3)$ & $<0.001$ \\
\hline ACE inhibitor/ARBs, n (\%) & $122(30.2)$ & $25(31.6)$ & $24(36.9)$ & $10(33.3)$ & 0.742 \\
\hline Calcium channel blocker, n (\%) & $63(15.6)$ & $20(25.3)$ & $13(20.0)$ & $5(16.7)$ & 0.198 \\
\hline Aspirin, n (\%) & $28(6.9)$ & $7(8.9)$ & $6(9.2)$ & $4(13.3)$ & 0.57 \\
\hline Statin, n (\%) & $44(10.9)$ & $9(11.4)$ & $8(12.3)$ & $4(13.3)$ & 0.968 \\
\hline $\mathrm{FPG}(\mathrm{mmol} / \mathrm{L})$ & $8.3 \pm 2.7$ & $8.4 \pm 2.8$ & $9.1 \pm 4.3$ & $9.4 \pm 1.5$ & 0.006 \\
\hline eGFR (mL/min/1.73 m²) & $86.7 \pm 16.1$ & $84.3 \pm 17.7$ & $81.1 \pm 20.6$ & $85.2 \pm 18.9$ & 0.042 \\
\hline Mean $\mathrm{HbA1c}(\%)$ & $8.1 \pm 1.5$ & $8.2 \pm 1.4$ & $9.2 \pm 1.6$ & $9.2 \pm 1.4$ & $<0.001$ \\
\hline $\mathrm{SD} \mathrm{HbA1c}$ & $1.1 \pm 1.0$ & $0.7 \pm 0.6$ & $1.3 \pm 1.3$ & $1.2 \pm 1.1$ & 0.285 \\
\hline Mean HbA1c (mmol/L) & $65.1 \pm 16.0$ & $64.8 \pm 16.3$ & $76.9 \pm 17.3$ & $77.4 \pm 15.6$ & $<0.001$ \\
\hline Mean total cholesterol (mmol/L) & $4.7 \pm 0.9$ & $4.7 \pm 0.9$ & $4.7 \pm 0.9$ & $5.2 \pm 0.8$ & 0.009 \\
\hline Mean triglyceride $(\mathrm{mmol} / \mathrm{L})$ & $1.7 \pm 1.0$ & $1.7 \pm 0.8$ & $1.6 \pm 0.9$ & $1.8 \pm 1.1$ & 0.971 \\
\hline Mean HDL-cholesterol (mmol/L) & $1.2 \pm 0.3$ & $1.1 \pm 0.3$ & $1.1 \pm 0.3$ & $1.2 \pm 0.3$ & 0.445 \\
\hline Mean LDL-cholesterol (mmol/L) & $2.7 \pm 0.8$ & $2.8 \pm 0.8$ & $2.8 \pm 0.9$ & $3.2 \pm 0.6$ & 0.003 \\
\hline UAE (mg/day) & $48.1 \pm 191.4$ & $142.5 \pm 481.0$ & $182.7 \pm 513.5$ & $120.4 \pm 137.9$ & 0.001 \\
\hline
\end{tabular}

Values are presented as number (\%) or mean \pm SD

CAN cardiovascular autonomic neuropathy, $A R B$ angiotensin receptor blocker, FPG fasting plasma glucose, eGFR estimated glomerular filtration rate, SD standard deviation, UAE urinary albumin excretion

heart rate variability [21]. Jaiswal et al. found that the prevalence of CAN in a young diabetes cohort was comparable to that reported in the adult diabetes population and that elevated triglyceride levels were the modifiable factors associated with CAN in this group [22]. Another recent report suggested that apolipoprotein A-1 level can be a useful marker for the presence of CAN [23].

In this study, the cumulative incidence of CAN was approximately $30 \%$, and $17.6 \%$ of patients in the progression group developed definite CAN over 2.3 years. The factors associated with CAN progression were age, diabetes duration, use of insulin, and increased HbA1c levels between baseline and follow-up CARTs, which were similar to findings from previous studies [24-26]. Patients with rapid CAN progression had higher glycemic and LDL-cholesterol levels than other patients, although other factors, including age, diabetes duration, presence of hypertension, BMI, and nephropathy, were not different between the patient groups. Poor glycemic control plays a central role in CAN progression, and intensive glycemic control and lipid modification can slow or delay the progression [27]. Hyperglycemia and dyslipidemia cause several metabolic pathways to enter a vicious cycle, resulting in the accumulation of toxic metabolic derivatives that contribute to neuronal damage and the development of micro- and macrovascular complications in type 2 diabetes $[3,28]$.

\section{Association between CAN progression and CVD}

CAN is a predictor of CVD and mortality in patients with type 2 diabetes. The Detection of Ischemia in Asymptomatic Diabetic Subjects study reported that CAN based on an abnormal Valsalva ratio test result was strongly associated with silent ischemia, independent of traditional CVD risk factors [14]. The Action to Control Cardiovascular Risk in Diabetes trial also showed that the presence of CAN was a significant predictive factor of cardiovascular mortality after adjustment for traditional CV risk factors [10]. We previously reported a significant association between CAN and ischemic stroke in a study of 1458 type 2 diabetes patients with a 7-year follow-up and between CAN and recurrent CVD in 206 type 2 diabetes patients with a 
Table 3 Crude and multivariable Cox proportional hazard model for cardiovascular disease and sensitive analysis for cardiovascular disease after exclusion of the patients who developed cardiovascular disease within 2 years

\begin{tabular}{|c|c|c|c|c|c|c|}
\hline & Crude HR (95\% Cl) & $P$ value & $\begin{array}{l}\text { Age and sex adjusted HR } \\
(95 \% \mathrm{Cl})\end{array}$ & $P$ value & $\begin{array}{l}\text { Fully adjusted HR } \\
(95 \% \mathrm{Cl})\end{array}$ & $P$ value \\
\hline \multicolumn{7}{|l|}{ Overall risk of CVD } \\
\hline Non-progression of CAN & Reference & & Reference & & Reference & \\
\hline Progression of CAN & $4.31(2.49-7.47)$ & $<0.001$ & $3.68(2.10-6.47)$ & $<0.001$ & $3.32(1.81-6.14)$ & $<0.001$ \\
\hline Non-progression & Reference & & Reference & & Reference & \\
\hline Normal to early & $2.73(1.28-5.81)$ & 0.009 & $2.30(1.07-4.94)$ & 0.032 & $2.68(1.19-6.02)$ & 0.017 \\
\hline Early to definite & $5.18(2.63-10.20)$ & $<0.001$ & $4.29(2.12-8.66)$ & $<0.001$ & $3.35(1.55-7.26)$ & 0.002 \\
\hline Normal to definite & $6.99(3.19-15.32)$ & $<0.001$ & $6.58(2.99-14.47)$ & $<0.001$ & $4.91(2.05-11.77)$ & $<0.001$ \\
\hline P for trend & $<0.001$ & & $<0.001$ & & 0.001 & \\
\hline \multicolumn{7}{|l|}{ Subgroup analysis } \\
\hline \multicolumn{7}{|l|}{ Age $<60$ years } \\
\hline Non-progression of CAN & Reference & & Reference & & Reference & \\
\hline Progression of CAN & $8.04(3.01-21.51)$ & $<0.001$ & $8.29(3.05-22.51)$ & $<0.001$ & $5.49(1.89-16.00)$ & 0.001 \\
\hline \multicolumn{7}{|l|}{ Age $\geq 60$ years } \\
\hline Non-progression of CAN & Reference & & Reference & & Reference & \\
\hline Progression of CAN & $2.59(1.33-5.03)$ & 0.005 & $2.46(1.25-4.81)$ & 0.009 & $2.29(1.10-4.77)$ & 0.03 \\
\hline \multicolumn{7}{|l|}{ Diabetes duration $<10$ years } \\
\hline Non-progression of CAN & Reference & & Reference & & Reference & \\
\hline Progression of CAN & $5.37(2.16-13.38)$ & $<0.001$ & $4.40(1.73-11.17)$ & 0.002 & $5.34(1.81-15.70)$ & 0.002 \\
\hline \multicolumn{7}{|l|}{ Diabetes duration $\geq 10$ years } \\
\hline Non-progression of CAN & Reference & & Reference & & Reference & \\
\hline Progression of CAN & $3.20(1.58-6.49)$ & 0.001 & $3.02(1.48-6.16)$ & 0.002 & $2.67(1.24-5.74)$ & 0.012 \\
\hline \multicolumn{7}{|l|}{$\mathrm{BMl}<25 \mathrm{~kg} / \mathrm{m}^{2}$} \\
\hline Non-progression of CAN & Reference & & Reference & & Reference & \\
\hline Progression of CAN & $5.28(2.43-11.47)$ & $<0.001$ & $4.86(2.22-10.64)$ & $<0.001$ & $4.87(2.00-11.90)$ & 0.001 \\
\hline \multicolumn{7}{|l|}{$\mathrm{BMI} \geq 25 \mathrm{~kg} / \mathrm{m}^{2}$} \\
\hline Non-progression of CAN & Reference & & Reference & & Reference & \\
\hline Progression of CAN & $3.70(1.66-8.26)$ & 0.001 & $2.81(1.22-6.48)$ & 0.016 & $3.39(1.38-8.37)$ & 0.008 \\
\hline \multicolumn{7}{|l|}{ Mean $\mathrm{HbA} 1 \mathrm{c}<9.0 \%$} \\
\hline Non-progression of CAN & Reference & & Reference & & Reference & \\
\hline Progression of CAN & $3.47(1.67-7.20)$ & 0.001 & $2.88(1.35-6.16)$ & 0.006 & $3.16(1.41-7.10)$ & 0.005 \\
\hline \multicolumn{7}{|l|}{ Mean $\mathrm{HbA} 1 \mathrm{c}<9.0 \%$} \\
\hline Non-progression of CAN & Reference & & Reference & & Reference & \\
\hline Progression of CAN & $4.47(1.86-10.72)$ & 0.001 & $3.91(1.61-0.95)$ & 0.003 & $5.44(2.01-14.72)$ & 0.001 \\
\hline \multicolumn{7}{|l|}{ Sensitive analysis } \\
\hline Non-progression of CAN & Reference & & Reference & & Reference & \\
\hline Progression of CAN & $3.41(1.81-6.43)$ & $<0.001$ & $2.94(1.54-5.63)$ & 0.001 & $2.85(1.40-5.78)$ & 0.004 \\
\hline
\end{tabular}

CVD cardiovascular disease, CAN cardiovascular autonomic neuropathy

9-year follow-up $[13,29]$. Progressive stages of CAN are commonly associated with increasingly worse prognosis $[6,7]$. However, there is limited evidence regarding the effect of CAN progression on CVD event. In this study, CAN progression was a significant predictor of CVD, and rapid CAN progression stage (normal to definite within 3 years) showed the highest risk for CVD development.

Several explanations are possible for the higher CVD risk in patients with CAN progression. Individuals with
CAN have impaired exercise tolerance and may resume exercise because of deteriorating cardiac pain perception during increasing myocardial ischemia [30]. The initial development of CAN in patients with diabetes is characterized by the relative augmentation of cardiac sympathetic activity due to parasympathetic denervation, which can be measured with parameters utilized in this study [31]. Increased sympathetic activity may increase cardiac loading, finally leading to impaired heart function. Sympathetic augmentation associated with CAN 


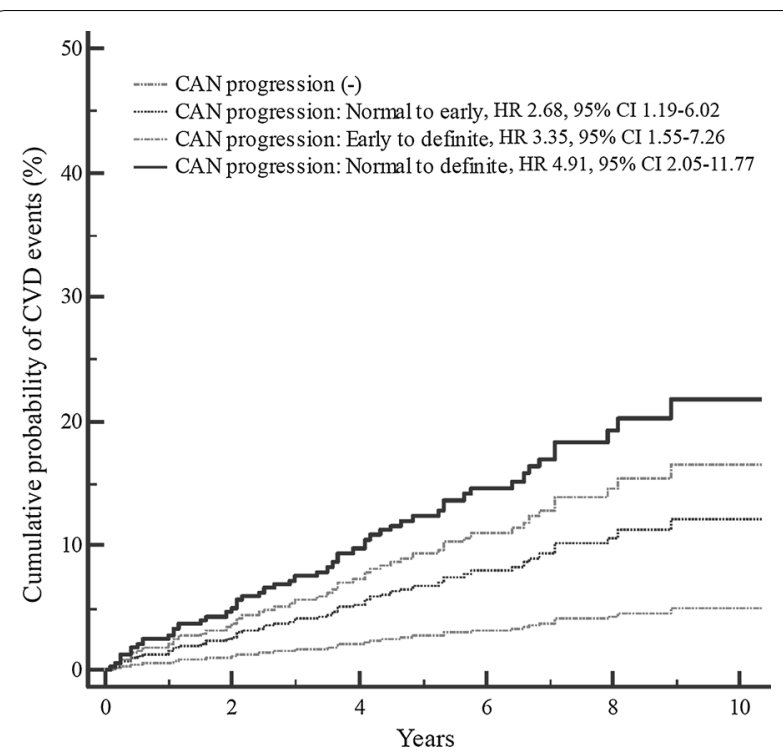

Fig. 1 Cumulative probability of cardiovascular events according to the progression status of cardiovascular autonomic neuropathy. CVD cardiovascular disease, CAN cardiovascular autonomic neuropathy

increases catecholamine levels, which causes a cytotoxic effect on the heart and contributes to myocardial damage associated with increased mitochondrial reactive oxygen species and apoptosis [32-34]. CAN also directly causes diastolic filling dysfunction and reduces left ventricle ejection fraction that can contribute to the development of cardiomyopathy and cardiac dysfunction, subsequently leading to an increased risk of CVD and mortality $[35,36]$. In contrast, fewer studies have assessed the association between CAN and cerebrovascular disease. Autonomic imbalance may alter cerebral regulation and variability in cerebral blood flow regulation, leading to overt cerebrovascular events [37]. In our study, the risk of CVD in the group with rapid CAN progression was higher than that in the group with consistently maintained definite CAN from baseline to follow-up CARTs. Rapid CAN progression might have more detrimental effects on the cardiovascular system, resulting in poorer outcomes $[1,6]$. In addition, patients with definite CAN at baseline CARTs received more insulin, blood pressure medications, and statin treatment than those with normal or early CAN.

There are other possible candidates, including glycemic variability, that can explain the interrelation mechanisms among metabolic disorder, rapid CAN progression, and CVD. Glycemic variability triggers oxidative stress as sustained hyperglycemia and affects the CVD event in association with insulin resistance or cellular metabolic memory $[20,38]$. CAN progression may also affect glycemic variability because the pancreatic $\beta$-cell is heavily innervated by parasympathetic fibers that stimulate $\beta$ cells to release insulin. However, in our study, standard deviation in HbA1c levels did not have a significant difference in predicting CVD. Further studies are needed to clarify this interrelationship.

\section{Effect modification for the association between CAN and CVD}

In this study, the magnitude of the risk of developing CVD was much higher in younger aged individuals, those with a shorter diabetes duration, or those with a lower BMI than in older patients, those with a longer diabetes duration, or those with a higher BMI. The former patients had a lower risk of CVD. In these patients, CAN progression may have a much greater impact on CVD development than other traditional CVD risk factors. In contrast, although poor glycemic control patients already have more risk factors for CVD, the effect of CAN progression on CVD was enhanced in patients with a poor glycemic status. This result demonstrated that poor glycemic control can have additional augmented effects on the association between CAN progression and CVD. The effect modification of dyslipidemia on the association between CAN progression and CVD was not shown in this study. However, it is difficult to explain the detailed mechanism of the effect modifications, and further analysis is required to clarify the issue.

\section{Limitations and strengths of this study}

This study had the following limitations. First, we mainly focused on the parasympathetic function of CARTs. However, these tests, which included heart rate response to deep breathing, Valsalva maneuver, and postural change, are widely recommended because of their high reliability and reproducibility [8]. We also assessed $\mathrm{BP}$ response to postural change. However, only $1.9 \%$ of enrolled patients progressed to severe CAN (presence of orthostatic hypotension), and we could not determine any significant results using this patient group. Second, the number of outcomes was small $(9.8 \%$ of total patients), and we could not perform subgroup analysis for coronary artery disease or cerebrovascular disease outcomes. Third, we did not assess baseline heart function or inflammatory biomarkers, which could affect the association between CAN and CVD in this study. Fourth, this study was conducted in one Asian ethnic group and one hospital-based cohort. More validation studies are needed to generalize the main hypothesis of this study. However, to the best of our knowledge, this is the first long-term, follow-up study that evaluated the association between CAN progression and CVD. We used mean HbA1c and LDL-cholesterol levels between baseline and follow-up CARTs to represent the more exact baseline glycemic and lipid status of patients. 


\section{Conclusion}

In summary, we suggest that CAN progression is an independent prognostic factor for CVD. Moreover, in our study, type 2 diabetes patients with rapid CAN progression had the greatest risk of CVD development. Rapid CAN progression was associated with poor glycemic control and poor LDL-cholesterol levels and regular screening for CAN is important to determine CVD risk. However, additional studies are required to clarify the precise mechanisms underlying the association between CAN progression and CVD and to apply these findings to other cohort or ethnic groups.

\section{Additional files}

Additional file 1: Figure S1. Study design summarization of the sample recruitment and follow-up. CART, cardiovascular autonomic reflex test; CVD, cardiovascular disease

Additional file 2: Table S1. Screening intervals according to the CAN status. Table S2. Descriptive characteristics according to the progression status of cardiovascular autonomic neuropathy including the group with definite CAN. Table S3. Crude and multivariable Cox proportional hazard model for cardiovascular disease and sensitive analysis for cardiovascular disease after exclusion of the patients who developed cardiovascular disease within 2 years.

\section{Abbreviations}

ARB: angiotensin II receptor blocker; CAN: cardiovascular autonomic neuropathy; CARTs: cardiovascular autonomic reflex tests; CVD: cardiovascular disease; HDL: high-density lipoprotein; LDL: low-density lipoprotein.

\section{Authors' contributions}

J-SY wrote the manuscript and interpreted data. Y-MP analyzed data. S-AC contributed to discussions. Y-BA reviewed the manuscript. Se-HK designed the study, collected and researched data, interpreted data, and drafted the manuscript. Se-HK is the guarantor of this work and, as such, had full access to all the data in this study and takes responsibility for the integrity and accuracy of the data. All authors read and approved the final manuscript.

\section{Author details}

${ }^{1}$ Division of Endocrinology and Metabolism, Department of Internal Medicine, St. Vincent's Hospital, College of Medicine, The Catholic University of Korea, Ji-dong, Paldal-gu, Suwon 16247, South Korea. ${ }^{2}$ Epidemiology Branch, National Institute of Environmental Health Sciences, National Institutes of Health, Research Triangle Park, Durham, NC, USA.

\section{Acknowledgements}

The authors thank E. Y. Kim, M. Shim, K. Shin, and Y. Yong (St. Vincent's Hospital, College of Medicine. The Catholic University of Korea) for their assistance collecting data for this manuscript.

\section{Competing interests}

The authors declare that they have no competing interests.

\section{Availability of data and materials}

The datasets used and/or analyzed in the current study are available from the corresponding author upon reasonable request.

\section{Consent for publication}

Not applicable.

\section{Ethics approval and consent to participate}

This study was approved by the Ethics Committee of Catholic Medical Center and was performed according to the Declaration of Helsinki.

\section{Funding}

Not applicable.

\section{Publisher's Note}

Springer Nature remains neutral with regard to jurisdictional claims in published maps and institutional affiliations.

Received: 17 May 2018 Accepted: 26 July 2018

Published online: 02 August 2018

References

1. Vinik Al, Ziegler D. Diabetic cardiovascular autonomic neuropathy. Circulation. 2007;115(3):387-97.

2. Pop-Busui R. Cardiac autonomic neuropathy in diabetes: a clinical perspective. Diab Care. 2010;33(2):434-41.

3. Kuehl M, Stevens MJ. Cardiovascular autonomic neuropathies as complications of diabetes mellitus. Nat Rev Endocrinol. 2012;8(7):405-16.

4. Maser RE, Lenhard MJ. Cardiovascular autonomic neuropathy due to diabetes mellitus: clinical manifestations, consequences, and treatment. J Clin Endocrinol Metabol. 2005;90(10):5896-903.

5. Fisher VL, Tahrani AA. Cardiac autonomic neuropathy in patients with diabetes mellitus: current perspectives. Diab Metab Syndr Obes. 2017:10:419-34.

6. Tesfaye S, Boulton AJ, Dyck PJ, Freeman R, Horowitz M, Kempler P, Lauria G, Malik RA, Spallone V, Vinik A, et al. Diabetic neuropathies: update on definitions, diagnostic criteria, estimation of severity, and treatments. Diab Care. 2010:33(10):2285-93.

7. Spallone V, Ziegler D, Freeman R, Bernardi L, Frontoni S, Pop-Busui R, Stevens M, Kempler P, Hilsted J, Tesfaye S, et al. Cardiovascular autonomic neuropathy in diabetes: clinical impact, assessment, diagnosis, and management. Diabetes/metabolism research and reviews. 2011;27(7):639-53.

8. Vinik Al, Maser RE, Mitchell BD, Freeman R. Diabetic autonomic neuropathy. Diab Care. 2003;26(5):1553-79.

9. Ewing DJ, Campbell IW, Clarke BF. Assessment of cardiovascular effects in diabetic autonomic neuropathy and prognostic implications. Ann Intern Med. 1980;92(2 Pt 2):308-11.

10. Pop-Busui R, Evans GW, Gerstein HC, Fonseca V, Fleg JL, Hoogwerf BJ, Genuth S, Grimm RH, Corson MA, Prineas R. Effects of cardiac autonomic dysfunction on mortality risk in the action to control cardiovascular risk in diabetes (ACCORD) trial. Diab Care. 2010;33(7):1578-84.

11. Yun JS, Ahn YB, Song KH, Yoo KD, Kim HW, Park YM, Ko SH. The association between abnormal heart rate variability and new onset of chronic kidney disease in patients with type 2 diabetes: a 10-year follow-up study. Diab Res Clin Pract. 2015;108(1):31-7.

12. Yun JS, Kim JH, Song KH, Ahn YB, Yoon KH, Yoo KD, Park YM, Ko SH. Cardiovascular autonomic dysfunction predicts severe hypoglycemia in patients with type 2 diabetes: a 10-year follow-up study. Diab Care 2014;37(1):235-41.

13. Cha SA, Yun JS, Lim TS, Min K, Song KH, Yoo KD, Park YM, Ahn YB, Ko $\mathrm{SH}$. Diabetic cardiovascular autonomic neuropathy predicts recurrent cardiovascular diseases in patients with type 2 diabetes. PLoS ONE. 2016;11(10):e0164807.

14. Wackers FJ, Young LH, Inzucchi SE, Chyun DA, Davey JA, Barrett EJ, Taillefer R, Wittlin SD, Heller GV, Filipchuk N, et al. Detection of silent myocardial ischemia in asymptomatic diabetic subjects: the DIAD study. Diab Care. 2004;27(8):1954-61.

15. Miettinen H, Lehto S, Salomaa V, Mahonen M, Niemela M, Haffner SM, Pyorala K, Tuomilehto J. Impact of diabetes on mortality after the first myocardial infarction. The FINMONICA Myocardial Infarction Register Study Group. Diab Care. 1998;21(1):69-75.

16. Boulton AJ, Vinik Al, Arezzo JC, Bril V, Feldman EL, Freeman R, Malik RA, Maser RE, Sosenko JM, Ziegler D. Diabetic neuropathies: a statement by the American Diabetes Association. Diab Care. 2005;28(4):956-62. 
17. Levey AS, Stevens LA, Schmid CH, Zhang YL, Castro AF 3rd, Feldman HI, Kusek JW, Eggers P, Van Lente F, Greene T, et al. A new equation to estimate glomerular filtration rate. Ann Intern Med. 2009;150(9):604-12.

18. Koivisto VA, Stevens LK, Mattock M, Ebeling P, Muggeo M, Stephenson J, Idzior-Walus B. Cardiovascular disease and its risk factors in IDDM in Europe. EURODIAB IDDM Complications Study Group. Diab Care. 1996;19(7):689-97.

19. Desouza CV, Bolli GB, Fonseca V. Hypoglycemia, diabetes, and cardiovascular events. Diab Care. 2010:33(6):1389-94.

20. Jun JE, Lee SE, Lee YB, Ahn JY, Kim G, Jin SM, Hur KY, Lee MK, Kim JH. Glycated albumin and its variability as an indicator of cardiovascular autonomic neuropathy development in type 2 diabetic patients. Cardiovasc Diabetol. 2017;16(1):127

21. Shimabukuro M, Tanaka A, Sata M, Dai K, Shibata Y, Inoue Y, Ikenaga H, Kishimoto S, Ogasawara K, Takashima A, et al. alpha-Glucosidase inhibitor miglitol attenuates glucose fluctuation, heart rate variability and sympathetic activity in patients with type 2 diabetes and acute coronary syndrome: a multicenter randomized controlled (MACS) study. Cardiovasc Diabetol. 2017;16(1):86.

22. Jaiswal M, Divers J, Urbina EM, Dabelea D, Bell RA, Pettitt DJ, Imperatore G, Pihoker C, Dolan LM, Liese AD, et al. Cardiovascular autonomic neuropathy in adolescents and young adults with type 1 and type 2 diabetes: the SEARCH for Diabetes in Youth Cohort Study. Pediatr Diab. 2018;19(4):680-9.

23. Chung JO, Park SY, Han JH, Cho DH, Chung DJ, Chung MY. Serum apolipoprotein A-1 concentrations and the prevalence of cardiovascular autonomic neuropathy in individuals with type 2 diabetes. J Diab Compl. 2018;32(4):357-61.

24. Low PA, Benrud-Larson LM, Sletten DM, Opfer-Gehrking TL, Weigand SD, O'Brien PC, Suarez GA, Dyck PJ. Autonomic symptoms and diabetic neuropathy: a population-based study. Diab Care. 2004;27(12):2942-7.

25. Pop-Busui R, Low PA, Waberski BH, Martin CL, Albers JW, Feldman EL, Sommer C, Cleary PA, Lachin JM, Herman WH. Effects of prior intensive insulin therapy on cardiac autonomic nervous system function in type 1 diabetes mellitus: the diabetes control and complications trial/epidemiology of diabetes interventions and complications study (DCCT/EDIC). Circulation. 2009;119(22):2886-93.

26. Valensi P, Paries J, Attali JR. Cardiac autonomic neuropathy in diabetic patients: influence of diabetes duration, obesity, and microangiopathic complications-the French multicenter study. Metab Clin Exp. 2003;52(7):815-20.

27. Gaede P, Vedel P, Parving HH, Pedersen O. Intensified multifactorial intervention in patients with type 2 diabetes mellitus and microalbuminuria: the Steno type 2 randomised study. Lancet. 1999;353(9153):617-22.

28. Boulton AJ, Malik RA, Arezzo JC, Sosenko JM. Diabetic somatic neuropathies. Diab Care. 2004;27(6):1458-86.

29. Ko SH, Song KH, Park SA, Kim SR, Cha BY, Son HY, Moon KW, Yoo KD, Park YM, Cho JH, et al. Cardiovascular autonomic dysfunction predicts acute ischaemic stroke in patients with Type 2 diabetes mellitus: a 7-year follow-up study. Diab Med. 2008;25(10):1171-7.

30. Shakespeare CF, Katritsis D, Crowther A, Cooper IC, Coltart JD, Webb-Peploe MW. Differences in autonomic nerve function in patients with silent and symptomatic myocardial ischaemia. Br Heart J. 1994;71(1):22-9.

31. Schonauer M, Thomas A, Morbach S, Niebauer J, Schonauer U, Thiele H. Cardiac autonomic diabetic neuropathy. Diab Vasc Dis Res. 2008;5(4):336-44

32. Paulson DJ, Light KE. Elevation of serum and ventricular norepinephrine content in the diabetic rat. Res Commun Chem Pathol Pharmacol. 1981:33(3):559-62.

33. Felten SY, Peterson RG, Shea PA, Besch HR Jr, Felten DL. Effects of streptozotocin diabetes on the noradrenergic innervation of the rat heart: a longitudinal histofluorescence and neurochemical study. Brain Res Bull. 1982;8(6):593-607.

34. Givertz MM, Sawyer DB, Colucci WS. Antioxidants and myocardial contractility: illuminating the "Dark Side" of beta-adrenergic receptor activation? Circulation. 2001;103(6):782-3.

35. Eichhorn EJ, Bristow MR. Medical therapy can improve the biological properties of the chronically failing heart. A new era in the treatment of heart failure. Circulation. 1996;94(9):2285-96.

36. Bristow MR. Mechanistic and clinical rationales for using beta-blockers in heart failure. J Cardiac Fail. 2000;6(2 Suppl 1):8-14.

37. Morita-Tsuzuki Y, Hardebo JE, Bouskela E. Interaction between cerebrovascular sympathetic, parasympathetic and sensory nerves in blood flow regulation. J Vasc Res. 1993;30(5):263-71.

38. Gorst C, Kwok CS, Aslam S, Buchan I, Kontopantelis E, Myint PK, Heatlie G, Loke Y, Rutter MK, Mamas MA. Long-term glycemic variability and risk of adverse outcomes: a systematic review and meta-analysis. Diab Care. 2015;38(12):2354-69.
Ready to submit your research? Choose BMC and benefit from:

- fast, convenient online submission

- thorough peer review by experienced researchers in your field

- rapid publication on acceptance

- support for research data, including large and complex data types

- gold Open Access which fosters wider collaboration and increased citations

- maximum visibility for your research: over $100 \mathrm{M}$ website views per year

At $\mathrm{BMC}$, research is always in progress.

Learn more biomedcentral.com/submissions 